\title{
Terahertz wave amplification by a laser-modulated relativistic electron beam
}

\author{
Hossein Saberi॰," Fazel Jahangiri®, and Ali Reza Niknam® \\ Laser and Plasma Research Institute, Shahid Beheshti University, 1983969411 Tehran, Iran
}

(Received 4 July 2021; accepted 16 December 2021; published 28 December 2021)

\begin{abstract}
Generation of tunable terahertz radiation based on the interaction of a frequency-chirped low power terahertz wave with a relativistic electron beam is proposed. The electron beam is first modulated temporally due to the ponderomotive force induced by the beat wave of two copropagating intense laser beams of slightly different frequencies. Then the electron beam interacts with a low-amplitude driver wave at terahertz frequency with a linear frequency chirping. A nonlinear transverse current density is driven that emits terahertz radiation at an up-shifted frequency with a relatively enhanced amplitude. The modulation process is analyzed theoretically by a complete set of fluid equations and considering the effects of both the electrons space-charge potential and the beat wave ponderomotive force. The initial density and energy of the electron beam are both optimized in order to improve the terahertz amplification process. The effect of the driver's frequency chirping, as well as the effects of the initial electron beam density and energy on the emitted terahertz radiation are investigated numerically. It is shown that the frequency chirping of the driver wave plays a key role in terahertz amplification. It is shown that there is an optimum chirp value for which the terahertz radiation is amplified significantly compared with the case without chirping. Moreover, transverse effects of the laser beams and the driver wave on the emitted terahertz radiation are also investigated.
\end{abstract}

DOI: 10.1103/PhysRevAccelBeams.24.120703

\section{INTRODUCTION}

Terahertz radiation with widespread applications in science, technology, and medicine has attracted many scientists in the past few decades [1-4]. Currently, low power terahertz sources are based on the interaction of ultrashort laser pulses with semiconductors [5-7], nonlinear crystals [8,9], and plasmas [10-14]. On the other side, accelerator based sources are playing a pivotal role toward high power terahertz radiation [15-21]. An electron beam with a regularly spaced density at intervals in the order of the terahertz wavelengths can be a source of coherent terahertz radiation $[20,21]$.

Utilizing laser beams for manipulation of the electron beam, i.e., rearranging the particles distribution in the phase space, and modulating the electron beam density, has become widespread recently [22-31]. These techniques are mainly based on modulator-chicane modules, in which the electron beam is sinusoidally modulated during the interaction with a laser beam inside an undulator and then

\footnotetext{
*ho_saberi@sbu.ac.ir
}

Published by the American Physical Society under the terms of the Creative Commons Attribution 4.0 International license. Further distribution of this work must maintain attribution to the author(s) and the published article's title, journal citation, and DOI. compressed longitudinally in a magnetic chicane. Various layouts have been proposed so far to improve the modulation process for generation of short-wavelength radiation including the combinations of one modulator-one chicane $[23,24]$, two modulators-one chicane [25], and two modulators-two chicanes [26,27], which are mainly used for the generation of short-wavelength radiation.

There are some schemes that employ two laser beams for the electron beam microbunching and terahertz generation [26-34]. As variants of echo-enabled harmonic generation, some techniques have been proposed for electron beam density modulation at terahertz frequency using two lasers with different frequencies [26-29]. A different combination of the undulator and chicane has been proposed by using two lasers with identical wavelength [30]. The generation of frequency-chirped density modulated electron beam in two modulators has also been studied, where two laser pulses with the same central frequencies but different chirp parameters interact sequentially with the electron beam in modulators. The modulated electron beam then is used to produce ultrashort terahertz radiation in a tapered undulator [31]. Electron beam microbunching using a two-frequency laser beam has also been studied theoretically by ignoring the space-charge effects, but taking it into account in numerical calculations [32]. In a different approach, a mechanism of using the beat wave of two laser beams with different frequencies has been proposed to create 
electron density modulation at terahertz frequency, while the space-charge effects of the electron beam were neglected $[33,34]$. The modulated electron beam then emits radiation at terahertz frequency when interacts with a wiggler magnetic field [33] or surface plasma wave [34]. In some schemes, a short laser pulse is used to produce a train of attosecond electron bunches [35-38] for generation of short x-ray radiation [35]. Attosecond electron bunch generation via ponderomotive force of two femtosecond lasers at different frequencies has been demonstrated experimentally [36]. In a different scheme, generation of short electron pulse trains in the interaction of laser field with a plasma mirror has been investigated numerically [37]. Femtosecond terahertz radiation from femtoslicing by a short laser pulse has been also demonstrated at BESSY [38].

In this paper, a uniform density electron beam is modulated with the ponderomotive force induced by the beat wave of two laser beams at slightly different frequencies. The electron beam modulation is analyzed theoretically by the complete set of fluid equations [39] and considering the effects of both the electrons space-charge potential and the laser beat wave ponderomotive force. Thereafter, the modulated electron beam interacts with a chirped-frequency low-amplitude driver wave at terahertz frequency that drives a nonlinear transverse current of electrons. Such frequency-chirped terahertz waves could be practically generated by employing some modified photomixing schemes $[40,41]$. The nonlinear current then emits terahertz radiation at a frequency higher than that of the driver and with a relatively improved amplitude. The frequency up-shift is of the order of the ponderomotive beat wave frequency. It is revealed that the terahertz radiation can be significantly enhanced by a resonantly chirped driver wave when compared to the case without frequency chirping.

The paper is organized as follows: In Sec. II, the theoretical model is described. In Sec. III, the results of the numerical calculations are presented and discussed. The effect of the driver's frequency chirping, as well as the effects of the electron beam parameters (i.e., density and velocity) are investigated numerically. In Sec. IV, some concluding remarks are made.

\section{THEORETICAL MODEL DESCRIPTION}

As shown in Fig. 1, two laser beams of different frequencies co-propagating along the $z$-axis interfere and create a beat wave, that then interacts with a relativistic electron beam moving along the lasers propagation axis. It causes the longitudinal density modulation of the electrons when passing through a drift space [33]. Thereafter, the density modulated electron beam interacts with a low intensity driver wave at terahertz frequency with a temporally varying frequency. The electron bunches are expected to interact with an almost fixed intensity of the driver wave around its focal region by considering a large enough radius for the terahertz wave to provide a long Rayleigh range. Terahertz mirrors have small apertures to let the electron beam with a much smaller size go through without considerable effect on the spatial distribution of the terahertz waves. As a result, a nonlinear transverse current is driven that emits terahertz radiation at a frequency higher than that of the driver. The frequency up-shift is of the order of the electron beam modulation, and hence the beat wave frequency. A resonantly chirped driver wave can enhance the terahertz radiation significantly compared with the case without chirping.

Laser beams are described as two linearly polarized plane waves propagating along the $z$-axis with the vector potential as follows:

$$
\mathbf{A}_{j}(z, t)=\hat{x} A_{j} e^{-i\left(\omega_{j} t-k_{j} z\right)}
$$

where $A_{j}$ is the constant amplitude of laser beams, $\omega_{j}$ and $k_{j}$ are the frequency and wave number of lasers, respectively, and $j=1,2$ denotes the laser beams numbers. The electric and magnetic fields of each laser are obtained as $\mathbf{E}_{j}=-\partial \mathbf{A}_{j} / \partial t$ and $\mathbf{B}_{j}=\nabla \times \mathbf{A}_{j}$, respectively. The electron beam with length $l_{\mathrm{b}}$ is assumed to have initial uniform

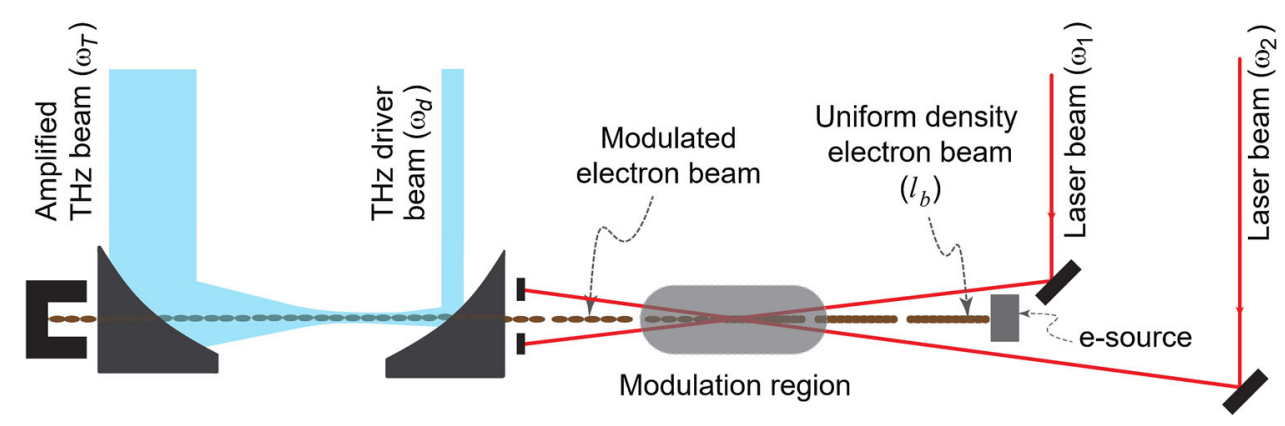

FIG. 1. The proposed layout of the interaction. The electron beam is modulated by the ponderomotive force induced by the lasers beat wave. Whereafter, the modulated electron beam interacts with a chirped-frequency low-amplitude driver wave at terahertz frequency that drives a nonlinear transverse current of electrons. The nonlinear oscillations of electrons will then emit high-intensity terahertz radiation. 
density $n_{0}$ and momentum $\mathbf{p}_{0}=\gamma_{0} m_{\mathrm{e}} \mathbf{v}_{0}$, where $m_{\mathrm{e}}$ denotes the electron mass, $\gamma_{0}=\left[1-v_{0}^{2} / c^{2}\right]^{-1 / 2}$ is the Lorentz factor, $\mathbf{v}_{0}=v_{0} \hat{z}$ is the initial electron beam velocity and $c$ is the speed of light in vacuum. The electron beam interacts with lasers beat wave.

The beat wave $|\mathbf{A}|^{2}=\left|\mathbf{A}_{1}+\mathbf{A}_{2}\right|^{2}$ has two characteristic phases corresponding to the sum and difference phases of the individual laser beams. In other words, the beat wave is characterized by a fast superluminal wave and a slow subluminal wave. As a result of the slow beat wave, lasers exert a ponderomotive force on the electrons. By neglecting fast oscillating terms, the ponderomotive force is given by [33]

$$
\mathbf{F}_{\text {pond }}=-\frac{e}{2}\left(\mathbf{v}_{1} \times \mathbf{B}_{2}^{*}+\mathbf{v}_{2}^{*} \times \mathbf{B}_{1}\right),
$$

where ${ }^{*}$ denotes the complex conjugate of the quantity. Here, $\mathbf{v}_{j}=e \mathbf{A}_{j} / \gamma_{0} m_{\mathrm{e}}$ is the oscillatory transverse velocity of the electron beam in the $j$ th laser beam, and $e$ is the electron charge. The ponderomotive force is then calculated as

$$
\mathbf{F}_{\text {pond }}=-\frac{i e^{2} k A_{1} A_{2}}{2 \gamma_{0} m_{\mathrm{e}}} e^{-i(\omega t-k z)} \hat{z},
$$

where $\omega=\omega_{1}-\omega_{2}$ and $k=k_{1}-k_{2}$ are the frequency and wave number of the slow beat wave, respectively. As a result of the axial ponderomotive force, the electrons will perform longitudinal space-charge oscillations at frequency $\omega$ that can be tuned over the terahertz frequency region, provided that proper lasers are chosen. Therefore, the velocity and, consequently, the density of the electrons would be modulated and electron bunches with regularly spaced density modulation in the order of the terahertz wavelength are produced. In the following section, by the complete set of fluid equations, a theoretical model of the electron beam modulation is presented.

\section{A. Electron beam modulation}

As the consequence of the laser beat wave interaction, the density, velocity, and the space-charge potential of the electron beam are perturbed as $n=n_{0}+n_{1}, \mathbf{v}=\mathbf{v}_{0}+\mathbf{v}_{1}$ and $\phi=\phi_{\mathrm{sc}}$, respectively, where $n_{1}$ and $\mathbf{v}_{1}=v_{1} \hat{z}$ are the perturbed quantities. In the fluid model, the electron beam behavior is described by

$$
\begin{gathered}
\frac{\partial \mathbf{p}}{\partial t}+\mathbf{v} \cdot \nabla \mathbf{p}=e \nabla \phi_{\mathrm{sc}}+\mathbf{F}_{\mathrm{pond}}, \\
\frac{\partial n}{\partial t}+\nabla \cdot(n \mathbf{v})=0, \\
\nabla^{2} \phi_{\mathrm{sc}}=\frac{e n_{1}}{\epsilon_{0}},
\end{gathered}
$$

which are the momentum $\left(\mathbf{p}=\gamma m_{\mathrm{e}} \mathbf{v}\right.$ with $\gamma=[1-$ $\left.v^{2} / c^{2}\right]^{-1 / 2}$ ), continuity and the Poisson equations, res- pectively. Equation (4) includes the effects of both the space-charge potential $\phi_{\mathrm{sc}}$, and the ponderomotive force $\mathbf{F}_{\text {pond }}$ on the momentum change.

As a response to the ponderomotive force, the perturbed quantities of the electron beam i.e., $n_{1}, \mathbf{v}_{1}$ and $\phi_{\text {sc }}$ have the dependency $e^{-i(\omega t-k z)}$ that indicates oscillations at frequency $\omega$ and wave number $k$. The equations are linearized by taking $\gamma \approx \gamma_{0}+\left(v_{0} \gamma_{0}^{3} / c^{2}\right) v_{1}$ and $\gamma \mathbf{v} \approx \gamma_{0} v_{0}+\gamma_{0}^{3} \mathbf{v}_{1}$. After some straightforward calculations, the perturbed quantities in the dimensionless units are obtained as follows:

$$
\begin{gathered}
v_{1}=\frac{k}{\gamma_{0}^{3}\left(\omega-k v_{0}\right)}\left[-\phi_{\mathrm{sc}}+\frac{A_{1} A_{2}}{2 \gamma_{0}}\right], \\
n_{1}=\frac{k}{\omega-k v_{0}} v_{1}, \\
\phi_{\mathrm{sc}}=-\frac{n_{1}}{k^{2}}
\end{gathered}
$$

Here, the normalized units are employed as $\omega \rightarrow \omega / \omega_{\text {pe }}$, $k \rightarrow k c / \omega_{\mathrm{pe}}, n_{1} \rightarrow n_{1} / n_{0}, v_{0,1} \rightarrow v_{0,1} / c, \phi_{\mathrm{sc}} \rightarrow e \phi_{\mathrm{sc}} / m_{\mathrm{e}} c^{2}$ and $A_{j} \rightarrow e A_{j} / m_{\mathrm{e}} c$, where $\omega_{\mathrm{pe}}=\sqrt{n_{0} e^{2} / \epsilon_{0} m_{\mathrm{e}}}$ is the plasma frequency of the electron beam. After some algebraic calculation of Eqs. (7)-(9), the perturbed density of the electron beam is obtained as

$$
n_{1}=\frac{k^{2} A_{1} A_{2}}{2 \gamma_{0}\left[\gamma_{0}^{3}\left(\omega-k v_{0}\right)^{2}-1\right]} e^{-i(\omega t-k z)} .
$$

A resonant density modulation will be achieved if $v_{0} \approx \omega / k$ similar to the Cerenkov resonance condition. The electron beam microbunching can be improved by using a magnetic chicane [22], or a drift space behind the modulator $[33,34,42]$ which is not discussed in this paper. The interaction of a chirped-frequency low-amplitude driver wave at terahertz frequency with the modulated electron beam drives a nonlinear transverse current of electrons that will be discussed in the next section.

\section{B. Nonlinear transverse current}

The chirped-frequency terahertz driver with the central frequency $\omega_{\mathrm{d}}$, wave number $k_{\mathrm{d}}$ and constant low-amplitude $A_{\mathrm{d}}$ is assumed to propagate along the $z$-axis and interact with the density-modulated electron beam. The vector potential of the driver wave is given by

$$
\mathbf{A}_{\mathrm{d}}(z, t)=\hat{x} A_{\mathrm{d}} e^{-i \Phi_{\mathrm{d}}(z, t)} .
$$

The instantaneous frequency of the driver is determined by the temporal variation of its phase $\Phi_{\mathrm{d}}(z, t)$. Here we consider a quadratic phase variation as 


$$
\Phi_{\mathrm{d}}(z, t)=\omega_{\mathrm{d}} t+b_{\mathrm{d}} t^{2}-k_{\mathrm{d}} z
$$

and, consequently, a linear frequency chirping as $\omega(t)=$ $\omega_{\mathrm{d}}+2 b_{\mathrm{d}} t$. Here, $b_{\mathrm{d}}$ denotes the chirp parameter, which can be considered positive or negative. The magnetic field of the driver wave is neglected as it is a low-amplitude wave, but, there is an electric field along the polarization direction. Therefore, the driver does not exert any longitudinal force on the electron beam, however, it causes transverse oscillations of the electrons along the electric field direction. The transverse motion is then described by the linearized transverse momentum equation as

$$
\gamma_{0} m_{\mathrm{e}}\left[\frac{\partial v_{x}}{\partial t}+\left(v_{0} \hat{z} \cdot \nabla\right) v_{x}\right]=-e \mathbf{E}_{\mathrm{d}}
$$

where the electric field is given by $\mathbf{E}_{\mathrm{d}}=-\partial \mathbf{A}_{\mathrm{d}} / \partial t$.

As a response of electrons to the driver wave, the transverse velocity along the $x$-axis has the dependency of $v_{x} \propto e^{-i \Phi_{\mathrm{d}}(z, t)}$. Thus, after a simple calculation we obtain the transverse velocity in the dimensionless units as

$$
v_{x}=\frac{\omega_{\mathrm{d}}+2 b_{\mathrm{d}} t}{\gamma_{0}\left(\omega_{\mathrm{d}}+2 b_{\mathrm{d}} t-k_{\mathrm{d}} v_{0}\right)} \mathbf{A}_{\mathrm{d}},
$$

where the chirp parameter is normalized as $b_{\mathrm{d}} \rightarrow b_{\mathrm{d}} / \omega_{\mathrm{pe}}^{2}$.

The longitudinal density perturbation, Eq. (10), in combination with the transverse velocity of the electron beam, Eq. (14), drives a nonlinear transverse current of electrons which is given by $\mathbf{J}_{\perp} \equiv \mathbf{J}^{\mathrm{NL}}=-e n_{1} v_{x}$. Substituting the perturbed quantities, the nonlinear current density in dimensionless units is obtained as

$$
\mathbf{J}^{\mathrm{NL}}=\hat{x} J^{\mathrm{NL}} e^{-i\left(\omega_{\mathrm{T}} t+b_{\mathrm{d}} t^{2}-k_{\mathrm{T}} z\right)},
$$

where

$J^{\mathrm{NL}}=-\frac{A_{1} A_{2} A_{\mathrm{d}}}{2 \gamma_{0}^{2}}\left[\frac{k^{2}}{\gamma_{0}^{3}\left(\omega-k v_{0}\right)^{2}-1}\right]\left[\frac{\omega_{\mathrm{d}}+2 b_{\mathrm{d}} t}{\omega_{\mathrm{d}}+2 b_{\mathrm{d}} t-k_{\mathrm{d}} v_{0}}\right]$,

in which $\omega_{\mathrm{T}}=\omega+\omega_{\mathrm{d}}, k_{\mathrm{T}}=k+k_{\mathrm{d}}$ and the current density is normalized as $\mathbf{J} \rightarrow \mathbf{J} / e n_{0} c$. This oscillating current emits electromagnetic light at frequency and wave number of $\omega_{\mathrm{T}}$ and $k_{\mathrm{T}}$, respectively. One can tune the nonlinear current density and, consequently, the frequency and amplitude of the emitted light by adjusting the beat wave frequency and amplitude as well as the frequency and chirp parameter of the driver wave. The phase term in Eq. (15) implies that the induced nonlinear current will also be frequency chirped, which leads to the generation of frequency-chirped terahertz waves. However, we ignore this phase term in the following calculations, because it could not affect the amplitude of the generated terahertz waves.

\section{Terahertz radiation}

The nonlinear current of the electron beam emits terahertz radiation with vector potential $\mathbf{A}_{\mathrm{T}}$. Therefore, the net electric and magnetic fields can be expressed in terms of the vector potential $\mathbf{A}=\mathbf{A}_{\mathrm{d}}+\mathbf{A}_{\mathrm{T}}$ and the scalar potential $\phi_{\text {sc }}$, as $\mathbf{E}=-\partial \mathbf{A} / \partial t-\nabla \phi_{\text {sc }}$ and $\mathbf{B}=\nabla \times \mathbf{A}$, respectively. By substituting the electric and magnetic fields into Ampere's law $\nabla \times \mathbf{B}=\mu_{0} \mathbf{J}+\partial \mathbf{E} / c^{2} \partial t$ we obtain

$$
\left(\frac{1}{c^{2}} \frac{\partial^{2}}{\partial t^{2}}-\nabla^{2}\right) \mathbf{A}=\mu_{0} \mathbf{J}-\frac{1}{c^{2}} \frac{\partial}{\partial t} \nabla \phi_{\mathrm{sc}}
$$

where the Coulomb gauge $\nabla \cdot \mathbf{A}=0$ is chosen. By a similar approach to [43] we separate current density into a transverse current $\mathbf{J}^{\mathrm{tr}}=\mathbf{J}^{\mathrm{d}}+\mathbf{J}^{\mathrm{NL}}$, which is associated with the electromagnetic waves (i.e., $\mathbf{J}^{\mathrm{d}}$ is the current density due to the terahertz driver wave and $\mathbf{J}^{\mathrm{NL}}$ is the nonlinear current), and a longitudinal current $\mathbf{J}^{\text {lon }}$ which is associated with the space-charge oscillations of the electron beam so that $\mathbf{J}=\mathbf{J}^{\text {tr }}+\mathbf{J}^{\text {lon }}$. After some straightforward calculations, the wave equation for the emitted light is obtained as

$$
\left(\frac{1}{c^{2}} \frac{\partial^{2}}{\partial t^{2}}-\nabla^{2}+\frac{1}{\gamma_{0}} \frac{\omega_{\mathrm{pe}}^{2}}{c^{2}}\right) \mathbf{A}_{\mathrm{T}}=\mu_{0} \mathbf{J}^{\mathrm{NL}},
$$

and the dispersion relation of the terahertz driver wave will be given as

$$
\omega_{\mathrm{d}}^{2}=k_{\mathrm{d}}^{2} c^{2}+\omega_{\mathrm{pe}}^{2} / \gamma_{0} .
$$

The generated light is expected to have the phase and polarization similar to the nonlinear current, so we may assume that

$$
\mathbf{A}_{\mathrm{T}}(z, t)=\hat{x} A_{\mathrm{T}}(z, t) e^{-i\left(\omega_{\mathrm{T}} t-k_{\mathrm{T}} z\right)},
$$

where the amplitude $A_{\mathrm{T}}(z, t)$ is a slowly varying function of $z$ and $t$ such that higher derivatives can be neglected. Therefore, the wave equation is simplified to

$\frac{\partial A_{\mathrm{T}}}{\partial z}+\frac{1}{v_{\mathrm{g}_{\mathrm{T}}}} \frac{\partial A_{\mathrm{T}}}{\partial t}+\frac{i}{2 k_{\mathrm{T}}}\left(k_{\mathrm{T}}^{2}-\frac{\omega_{\mathrm{T}}^{2}}{c^{2}}+\frac{1}{\gamma_{0}} \frac{\omega_{\mathrm{pe}}^{2}}{c^{2}}\right) A_{\mathrm{T}}=\frac{i \mu_{0} J^{\mathrm{NL}}}{2 k_{\mathrm{T}}}$,

where $v_{\mathrm{g}_{\mathrm{T}}}=k_{\mathrm{T}} c^{2} / \omega_{\mathrm{T}}$ is the group velocity of the emitted light. The third term in the left-hand side of Eq. (21) can be omitted under the perfect phase matching condition for the terahertz wave as $\omega_{\mathrm{T}}^{2}=c^{2} k_{\mathrm{T}}^{2}+\omega_{\mathrm{pe}}^{2} / \gamma_{0}$. Hence, The normalized wave equation is obtained as 


$$
\frac{\partial A_{\mathrm{T}}}{\partial z}+\frac{1}{\beta_{\mathrm{g}_{\mathrm{T}}}} \frac{\partial A_{\mathrm{T}}}{\partial t}=\frac{i J^{\mathrm{NL}}}{2 k_{\mathrm{T}}},
$$

where $\beta_{\mathrm{g}_{\mathrm{T}}}=v_{\mathrm{g}_{\mathrm{T}}} / c$ and $J^{\mathrm{NL}}$ is given by Eq. (16). Equation (22) is further simplified by using a new set of variables $\xi=z$ and $\psi=t-z / \beta_{\mathrm{g}_{\mathrm{T}}}$ to

$$
\frac{\partial A_{\mathrm{T}}}{\partial \xi}=\frac{i J^{\mathrm{NL}}}{2 k_{\mathrm{T}}} .
$$

We assume the interaction time to be $\tau_{\text {int }}$, and hence the interaction length will be $l_{\text {int }}=v_{0} \tau_{\text {int }}$. These quantities are normalized as $l_{\text {int }} \rightarrow l_{\text {int }} \omega_{\text {pe }} / c$ and $\tau_{\text {int }} \rightarrow \omega_{\text {pe }} \tau_{\text {int }}$. Equation (23) can be integrated over the interaction length to give the maximum amplitude of the emitted light normalized to the driver's amplitude as

$$
\begin{aligned}
\left|\frac{A_{\mathrm{T}}}{A_{\mathrm{d}}}\right|_{\max }= & \frac{A_{1} A_{2} l_{\mathrm{int}}}{4 k_{\mathrm{T}} \gamma_{0}^{2}}\left|\frac{k^{2}}{1-\gamma_{0}^{3}\left(\omega-k v_{0}\right)^{2}}\right| \\
& \times\left|\frac{\omega_{\mathrm{d}}+2 b_{\mathrm{d}} \tau_{\mathrm{int}}}{\omega_{\mathrm{d}}+2 b_{\mathrm{d}} \tau_{\mathrm{int}}-k_{\mathrm{d}} v_{0}}\right| .
\end{aligned}
$$

One can conclude from this equation that a higher terahertz amplification gain would be achieved by increasing the input laser intensities as well as the interaction length. It is also clear that Eq. (24) contains two resonances at which the amplitude of the emitted radiation could be maximized by properly choosing the initial electron beam velocity as well as the chirp parameter of the driver wave. The first resonance condition is achieved when $v_{0} \approx \omega / k$ that gives

$$
\left|\frac{A_{\mathrm{T}}}{A_{\mathrm{d}}}\right|_{\max } \approx \frac{A_{1} A_{2} l_{\mathrm{int}} k^{2}}{4 k_{\mathrm{T}} \gamma_{0}^{2}}\left|\frac{\omega_{\mathrm{d}}+2 b_{\mathrm{d}} \tau_{\mathrm{int}}}{\omega_{\mathrm{d}}+2 b_{\mathrm{d}} \tau_{\mathrm{int}}-k_{\mathrm{d}} v_{0}}\right| .
$$

It shows a dependency of $\left|A_{\mathrm{T}} / A_{\mathrm{d}}\right|_{\max } \propto k^{2}$ indicating that the emitted terahertz amplification gain increases at higher beat wave frequencies. The second resonance condition is due to the resonant chirp parameter, i.e., when the condition $\omega_{\mathrm{d}}+2 b_{\mathrm{d}} \tau_{\text {int }}-k_{\mathrm{d}} v_{0}=0$ is satisfied, and hence significant terahertz amplification is achieved. The resonant chirp parameter is obtained as

$$
b_{\mathrm{d}}^{\mathrm{res}}=-\frac{\omega_{\mathrm{d}}-k_{\mathrm{d}} v_{0}}{2 \tau_{\mathrm{int}}} .
$$

With carefully chosen parameters, the emitted light can be swept over the entire frequency range of the terahertz gap. In other words, with a given beat wave frequency $\omega$ and an arbitrary chosen terahertz frequency $\omega_{\mathrm{T}}$, the driver's frequency is determined by $\omega_{\mathrm{d}}=\omega_{\mathrm{T}}-\omega$. The electron beam critical density is then determined according to the terahertz driver wave frequency as $n_{\mathrm{cr}}=\epsilon_{0} m_{\mathrm{e}} \omega_{\mathrm{d}}^{2} / e^{2}$. In the next section, within some numerical calculation the terahertz amplification and the effect of the driver's frequency chirping are investigated, and the optimum parameters are obtained. Moreover, the effects of the initial electron beam density and energy will be investigated numerically to find the optimum electron beam parameters for the terahertz amplification.

\section{Transverse effects on terahertz generation}

The transverse effects of the laser beams and the driver wave are important and could influence the terahertz generation process. The laser beams are assumed to have transverse Gaussian profiles as

$$
\mathbf{A}_{j}(x, y, z ; t)=\hat{x} A_{j} e^{-\left(x^{2}+y^{2}\right) / w_{0}^{2}} e^{-i\left(\omega_{j} t-k_{j} z\right)},
$$

where $w_{0}$ is the spot size that is assumed to be identical. The ponderomotive force imposed by the laser beams is then calculated as

$$
\begin{aligned}
\mathbf{F}_{\text {pond }}= & -\frac{e^{2}}{2 \gamma_{0} m_{\mathrm{e}}}\left(i k \hat{z}-\frac{4 y}{w_{0}^{2}} \hat{y}\right) \\
& \times A_{1} A_{2} e^{-2\left(x^{2}+y^{2}\right) / w_{0}^{2}} e^{-i(\omega t-k z)},
\end{aligned}
$$

which includes an additional transverse component in the y-direction compared with that in Eq. (3). With some algebraic calculation of Eqs. (4)-(6) by using the ponderomotive force of Eq. (28), the normalized perturbed density of the electron beam is obtained as

$$
\begin{aligned}
n_{1}= & \frac{A_{1} A_{2}}{\gamma_{0}\left[\gamma_{0}^{3}\left(\omega-k v_{0}\right)^{2}-1\right]}\left[\frac{k^{2}}{2}+\frac{2}{w_{0}^{2}}\left(1-\frac{4 y^{2}}{w_{0}^{2}}\right)\right] \\
& \times e^{-2\left(x^{2}+y^{2}\right) / w_{0}^{2}} e^{-i(\omega t-k z)} .
\end{aligned}
$$

Similarly, to consider the transverse effect of the driver wave, a Gaussian profile in the transverse direction is considered such as

$$
\mathbf{A}_{\mathrm{d}}(x, y, z ; t)=\hat{x} A_{\mathrm{d}} e^{-\left(x^{2}+y^{2}\right) / w_{0}^{2}} e^{-i \Phi_{\mathrm{d}}},
$$

with a spot size similar to that of the laser beams. Then the transverse velocity of electrons in the $x$-direction is given by

$$
v_{x}=\frac{\omega_{\mathrm{d}}+2 b_{\mathrm{d}} t}{\gamma_{0}\left(\omega_{\mathrm{d}}+2 b_{\mathrm{d}} t-k_{\mathrm{d}} v_{0}\right)} A_{\mathrm{d}} e^{-\left(x^{2}+y^{2}\right) / w_{0}^{2}} e^{-i \Phi_{\mathrm{d}}} .
$$

It is obvious that Eqs. (29) and (31) well confirm those obtained by plane laser beams, i.e., Eqs. (10) and (14), respectively, when the spot sizes are considered large enough $\left(w_{0} \rightarrow \infty\right)$.

The amplitude of the nonlinear transverse current is modified as 


$$
\begin{aligned}
\mathcal{J}^{\mathrm{NL}}= & -\left[\frac{A_{1} A_{2} A_{\mathrm{d}}}{\gamma_{0}^{2}\left(\gamma_{0}^{3}\left(\omega-k v_{0}\right)-1\right)}\right]\left[\frac{\omega_{\mathrm{d}}+2 b_{\mathrm{d}} t}{\omega_{\mathrm{d}}+2 b_{\mathrm{d}} t-k_{\mathrm{d}} v_{0}}\right] \\
& \times\left\{\frac{k^{2}}{2}+\frac{2}{w_{0}^{2}}\left(1-\frac{4 y^{2}}{w_{0}^{2}}\right)\right\} e^{-3\left(x^{2}+y^{2}\right) / w_{0}^{2}} .
\end{aligned}
$$

Then the modified amplitude of the emitted terahertz wave including the transverse effects is given by

$$
\nabla_{\perp}^{2} \mathcal{A}_{\mathrm{T}}+2 i k_{\mathrm{T}} \frac{\partial \mathcal{A}_{\mathrm{T}}}{\partial z}=-\mathcal{J}^{\mathrm{NL}}
$$

where $\nabla_{\perp}^{2} \equiv \partial^{2} / \partial x^{2}+\partial^{2} / \partial y^{2}$. The transverse profile of terahertz radiation at an arbitrary distance along the $z$-axis is given by

$$
\nabla_{\perp}^{2} \mathcal{A}_{\mathrm{T}}(x, y)=-\mathcal{J}^{\mathrm{NL}} .
$$

\section{RESULTS AND DISCUSSION}

For consistency with any terahertz driver wave we consider the critical density of the electron beam for $f_{\mathrm{d}}=$ $0.1 \mathrm{THz}$ as $n_{\text {cr }} \equiv n_{0.1}=1.24 \times 10^{14} \mathrm{~cm}^{-3}$. Moreover, we choose $\mathrm{CO}_{2}$ laser beams for $9.3 \mu \mathrm{m}$ and $10.6 \mu \mathrm{m}$ lines, so that the slow beat wave's wavelength (frequency) will be $75.83 \mu \mathrm{m}(3.95 \mathrm{THz})$. Laser 1 is considered to have the normalized amplitude $A_{1}=3.67 \times 10^{-2}$ that corresponds to the intensity of $2 \times 10^{14} \mathrm{~W} / \mathrm{cm}^{2}$. Laser 2 has the normalized amplitude $A_{2}=4.79 \times 10^{-2}$ that corresponds to the intensity of $3 \times 10^{14} \mathrm{~W} / \mathrm{cm}^{2}$. Here, the normalized amplitudes are obtained as $A_{j}=0.85 \sqrt{I_{j_{18}} \lambda_{j}[\mu \mathrm{m}]}$, where $I_{j 18}$ is the laser intensity in the unit of $10^{18} \mathrm{~W} / \mathrm{cm}^{2}$, and $\lambda_{j}$ is the laser's wavelength in the unit of $\mu \mathrm{m}$. The initial electron beam density is $n_{0}=n_{0.1}$ that is uniform over its volume. The electron beam has an initial relativistic energy of $4 \mathrm{MeV}$.

For an arbitrary terahertz frequency, e.g., $f_{\mathrm{T}}=4.5 \mathrm{THz}$, the driver wave should be of frequency $f_{\mathrm{d}}=0.55 \mathrm{THz}$. The optimum chirp parameter of the terahertz driver is given by Eq. (26) as $b_{\mathrm{d}}^{\mathrm{opt}}=-5.35 \times 10^{-4}$ (in dimensionless units), which is slightly deviated from the resonance to avoid divergent numerical results.

We assume that the interaction length (and so the interaction time) of the driver wave and the electron beam is $l_{\text {int }}=2.5 \mathrm{~cm}$ (and $\tau_{\text {int }}=84 \mathrm{ps}$ ). With these parameters, the emitted light has the maximum normalized amplitude of $\left|A_{\mathrm{T}} / A_{\mathrm{d}}\right|_{\max }=36$. All the parameters and their values for the numerical calculations are summarized in Table I.

By adjusting the frequency of the beat wave, it could be possible to tune the frequency of the generated terahertz waves over the range of the terahertz gap. Figure 2 presents the maximum amplification gain versus frequency for terahertz waves generated by a beat wave with the frequency of $4.5 \mathrm{THz}$. In this figure, terahertz amplification gain obtained by the driver wave with optimal chirp value
TABLE I. All parameters and their values for the numerical calculation.

\begin{tabular}{lll}
\hline \hline Quantity & \multicolumn{1}{c}{ Symbol } & \multicolumn{1}{c}{ Value [Unit] } \\
\hline Electron beam & & \\
$\quad$ Energy & $E_{0}\left(=\gamma_{0} m_{\mathrm{e}} c^{2}\right)$ & $4[\mathrm{MeV}]$ \\
$\quad$ Density & $n_{0}\left(=n_{0.1}\right)$ & $1.24 \times 10^{14}\left[\mathrm{~cm}^{-3}\right]$ \\
Lasers in modulator & & \\
Laser 1 & & $9.3[\mu \mathrm{m}]$ \\
$\quad$ Wavelength & $\lambda_{1}$ & $50[\mu \mathrm{m}]$ \\
$\quad$ Spot size & $w_{0}$ & $3.67 \times 10^{-2}$ \\
$\quad$ Normalized amplitude & $A_{1}$ & $10.6[\mu \mathrm{m}]$ \\
Laser 2 & & $50[\mu \mathrm{m}]$ \\
$\quad$ Wavelength & $\lambda_{2}$ & $4.79 \times 10^{-2}$ \\
$\quad$ Spot size & $w_{0}$ & \\
$\quad$ Normalized amplitude & $A_{2}$ & $3.95[\mathrm{THz}]$ \\
Slow beatwave & & $75.83[\mu \mathrm{m}]$ \\
$\quad$ Frequency & $f(\omega=2 \pi f)$ & \\
$\quad$ Wavelength & $\lambda(k=2 \pi / \lambda)$ & $0.55[\mathrm{THz}]$ \\
Terahertz driver & & $50[\mu \mathrm{m}]$ \\
$\quad$ Frequency & $f_{\mathrm{d}}\left(\omega_{\mathrm{d}}=2 \pi f_{\mathrm{d}}\right)$ & $-5.35 \times 10^{-4}$ \\
$\quad$ Spot size & $w_{0}$ & $2.5[\mathrm{~cm}]$ \\
Chirp parameter & $b_{\mathrm{d}}^{\mathrm{opt}}$ & $84[\mathrm{ps}]$ \\
Interaction length & $l_{\text {int }}$ & $4.5[\mathrm{THz}]$ \\
Interaction time & $\tau_{\mathrm{int}}$ & 36 \\
Terahertz emission & & $f_{\mathrm{T}}\left(\omega_{\mathrm{T}}=2 \pi f_{\mathrm{T}}\right)$ \\
Frequency & $\left|A_{\mathrm{T}} / A_{\mathrm{d}}\right|_{\max }$ & \\
Relative amplitude & & \\
\hline \hline
\end{tabular}

$b_{\mathrm{d}}^{\text {opt }}$ has been compared with those of two slightly different chirp parameters (i.e., $b_{\mathrm{d}}^{( \pm)}=b_{\mathrm{d}}^{\mathrm{opt}} \pm 10^{-4}$ ), as well with the unchirped case. Here, the beat wave frequency is kept fixed

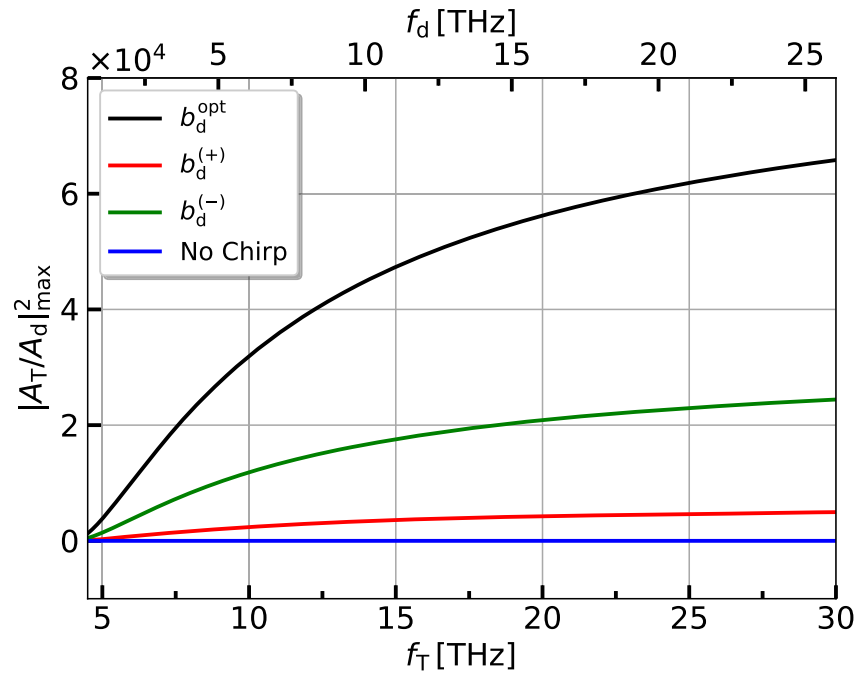

FIG. 2. Terahertz amplification gain versus its frequency for different chirp parameters, i.e., optimum chirp, $b_{\mathrm{d}}^{( \pm)}=b_{\mathrm{d}}^{\text {opt }} \pm$ $10^{-4}$ and the case without frequency chirping. The upper horizontal axis shows the driver wave's frequency. All parameters are the same as in Table I. 

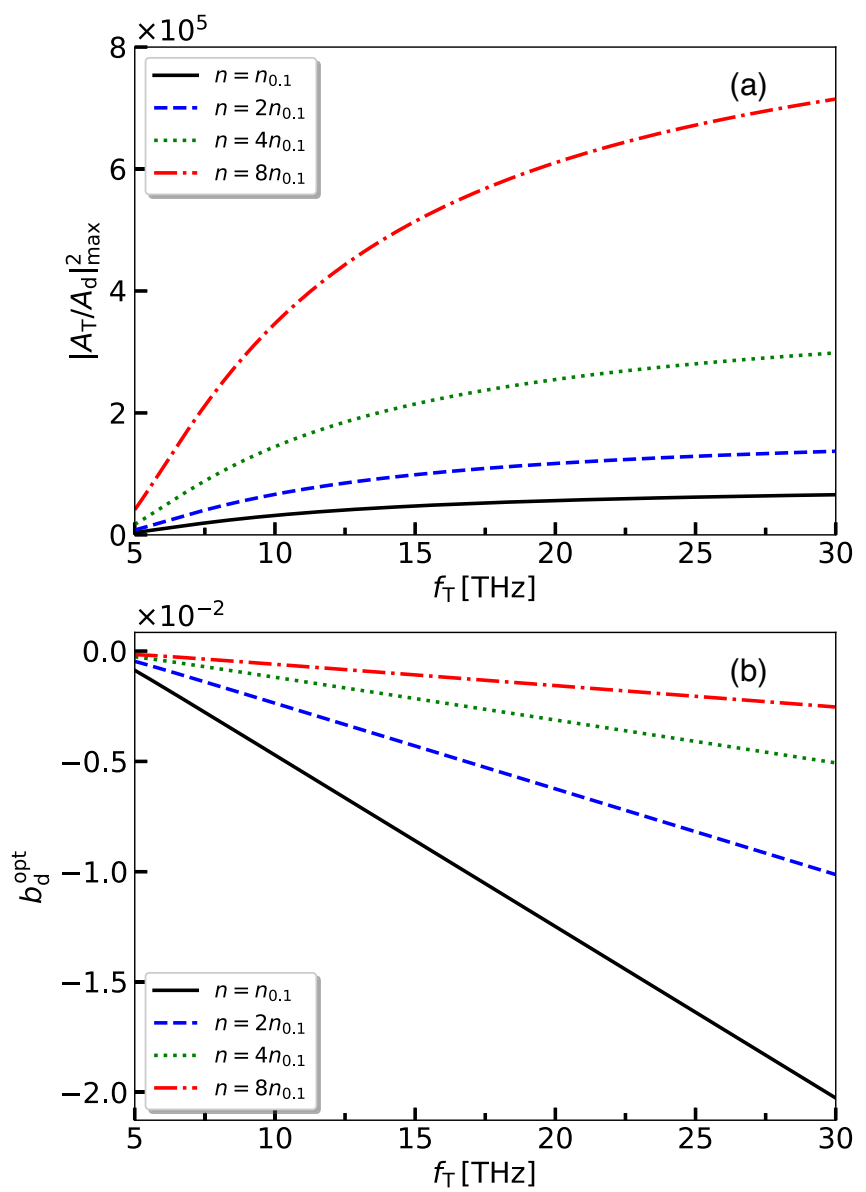

FIG. 3. Terahertz amplification gain (a), and the optimum frequency chirp parameter of the driver wave (b) versus frequency for different initial electron beam densities. All parameters are the same as in Table I.

according to that in Table I, but the driver wave frequency varies such that the terahertz emission will be tuned over the range of $4.5-30 \mathrm{THz}$. The driver wave frequency corresponding to each up-shifted terahertz frequency is also indicated in the plot. This figure shows that the driver wave chirp parameter has a large impact on the terahertz amplification process, especially when it is close enough to its resonance value. On the other hand, even a small deviation from the optimum chirp value would mitigate the terahertz amplification. Moreover, it can be seen in Fig. 2 that the amplification gain increases at higher terahertz frequencies.

The effect of the initial electron beam density on terahertz generation is explored in Figs. 3 and 4. Figure 3(a) compares the maximum amplification gain for different initial electron beam densities. It shows that as the density increases, a higher terahertz amplification gain is achieved. This result is in agreement with our intuition that larger number of oscillating electrons emit more intense radiation. As shown in Fig. 3(b), the absolute value of chirp parameter decreases as the density increases. The

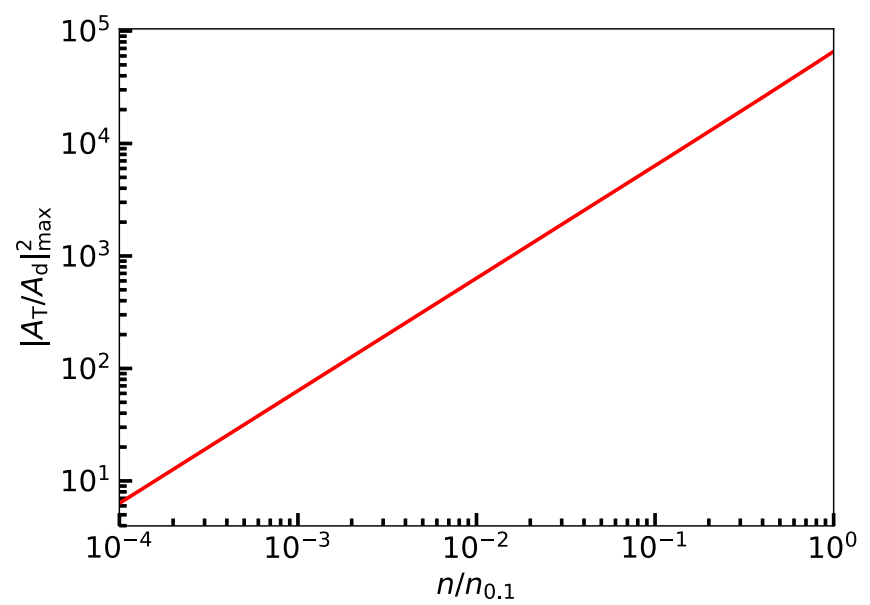

FIG. 4. Terahertz amplification gain versus the initial electron beam densities lower than the critical density for terahertz frequency $f_{\mathrm{T}}=30 \mathrm{THz}$. Other parameters are the same as in Table I.
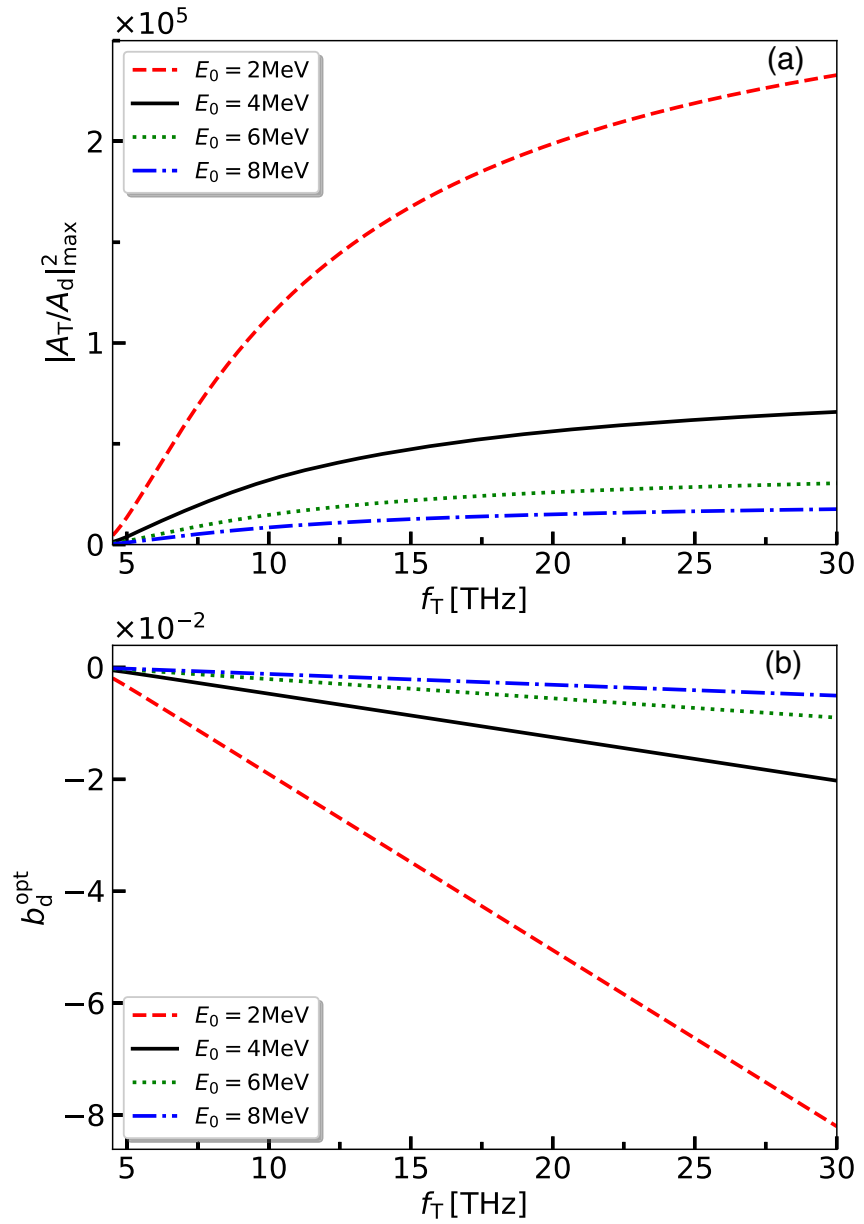

FIG. 5. Terahertz amplification gain (a), and the optimum frequency chirp parameter of the driver wave (b) versus frequency for different electron beam energies. All parameters are the same as in Table I. 


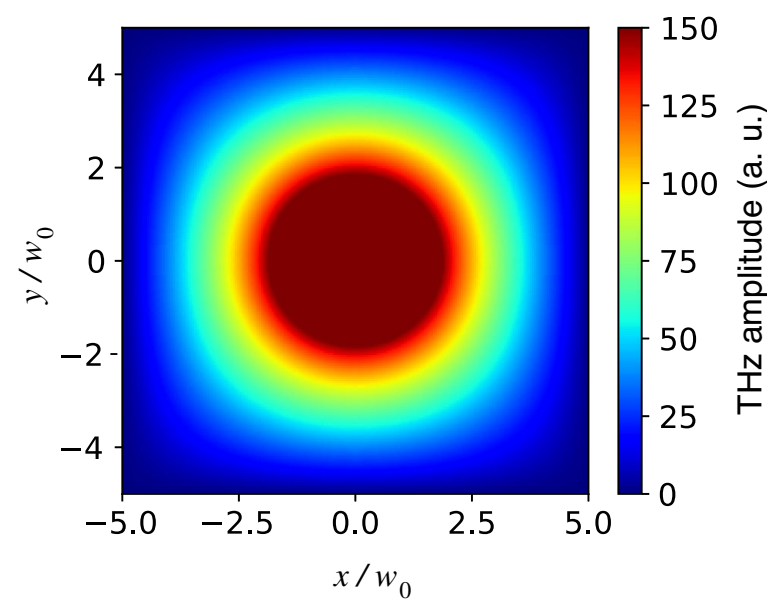

FIG. 6. Terahertz amplitude dependency on the transverse dimensions.

lower the absolute value of the chirp parameter, the easier to make it practically in the experiment. Therefore, an electron beam with high density would be preferable for terahertz amplification in this scheme. Figure 4 represents maximum terahertz amplification obtained at densities lower than the critical electron beam density. It shows that even with smaller densities that are more feasible in the experiment we could obtain reasonable amplification in this mechanism. Low electron beam densities, however, need higher chirp parameters that may exert a practical limitation on this mechanism [44]. Therefore, an optimum combination of electron beam density and chirp parameter of the driver wave should be used to generate high intensity terahertz wave at the chosen frequency.

As explained previously, in order to have a resonance density modulation, the initial electron beam velocity should be relativistic such that $v_{0}=\omega c / k \approx c$. Then we will have the dependency $\left|A_{\mathrm{T}} / A_{\mathrm{d}}\right|_{\max } \propto 1 / \gamma_{0}^{2}$, as can be seen in Eq. (25). Therefore, it is expected that the terahertz amplification decreases as the initial electron beam energy increases, which is verified in Fig. 5. The maximum amplification gain for different electron beam energies is compared in Fig. 5(a). It indicates that a low energy electron beam emits higher amplitude terahertz, however, lower relativistic electron beam needs terahertz driver wave with higher frequency chirping as shown in Fig. 5(b), that may be less practically feasible. In other words, one may conclude that with the electron beam of the energy around 2-4 MeV, higher terahertz amplification is achieved with a lower frequency chirping of the driver wave.

Figure 6 shows the transverse dependency of the emitted terahertz amplitude. It can be seen that the spatial distribution of the terahertz intensity is slightly asymmetric over the transverse dimension, which could be attributed to the appearance of the $y$-component of the ponderomotive force as in Eq. (28).

\section{CONCLUSIONS}

A mechanism of the terahertz radiation amplification by a laser-modulated relativistic electron beam interacting with a chirped-frequency low-amplitude driver wave at terahertz frequency was proposed. A uniform density relativistic electron beam has been modulated by the interaction with the beat wave of two lasers with different frequencies. The electron beam modulation has been analyzed theoretically by the complete set of fluid equations and considering the effects of both the electron beam space-charge potential and the lasers ponderomotive force. Thereafter, the modulated electron beam interacted with a co-propagating chirped-frequency terahertz driver wave that drives a nonlinear transverse current of electrons. The nonlinear current could emit electromagnetic light at frequencies higher than that of the driver. The frequency up-shift is in the order of the beat wave frequency. By a resonant chirp parameter of the driver, the terahertz radiation is amplified significantly compared with the case without chirping. Our numerical results suggest that higher terahertz amplification is achieved as the electron beam density increases. In addition, it has been shown that the mechanism works well with an electron beam of lower energies, though it requires higher less practically feasible chirp parameters for the driver wave. Moreover, numerical calculations have revealed that for a smaller and more practical chirp, higher terahertz amplification is achieved when the electron beam energy is about $2-4 \mathrm{MeV}$. We have proposed an alternative optical wiggler for terahertz wave emission from a laser-modulated electron beam based on utilizing a practically feasible chirped terahertz driver wave. It could provide a controllable high amplification gain and output bandwidth. It should be noted that the proposed scheme would require a high current electron beam which may be considered as a limitation.

[1] M. Tonouchi, Cutting-edge terahertz technology, Nat. Photonics 1, 97 (2007).

[2] X.C. Zhang, A. Shkurinov, and Y. Zhang, Extreme terahertz science, Nat. Photonics 11, 16 (2017).

[3] D. M. Mittleman, Perspective: Terahertz science and technology, J. Appl. Phys. 122, 230901 (2017).

[4] S.S. Dhillon et al., The 2017 terahertz science and technology roadmap, J. Phys. D 50, 043001 (2017).

[5] R. Khler, A. Tredicucci, F. Beltram, H. E. Beere, E. H. Linfield, A. G. Davies, D. A. Ritchie, R. C. Iotti, and F. Rossi, Terahertz semiconductor-heterostructure laser, Nature (London) 417, 156 (2002).

[6] G. P. Gallerano and S. Biedron, Overview of terahertz radiation sources, in Proceedings of 26th Free Electron Laser Conference, Trieste, Italy (2004) pp. 216-221.

[7] J. Shan and T. F. Heinz, Ultrafast Dynamical Processes in Semiconductors (Springer-Verlag Berlin Heidelberg, 2004) Chap. Terahertz Radiation from Semiconductors, pp. 1-59. 
[8] M. M. Nazarov, S. A. Makarova, A. P. Shkurinov, and O. G. Okhotnikov, The use of combination of nonlinear optical materials to control terahertz pulse generation and detection, Appl. Phys. Lett. 92, 021114 (2008).

[9] J. A. Flp, Z. Ollmann, C. Lombosi, C. Skrobol, S. Klingebiel, L. Plfalvi, F. Krausz, S. Karsch, and J. Hebling, Efficient generation of thz pulses with $0.4 \mathrm{mj}$ energy, Opt. Express 22, 20155 (2014).

[10] H. Hamster, A. Sullivan, S. Gordon, W. White, and R. W. Falcone, Subpicosecond, Electromagnetic Pulses from Intense Laser-Plasma Interaction, Phys. Rev. Lett. 71, 2725 (1993).

[11] G. Rodriguez and G. L. Dakovski, Scaling behavior of ultrafast two-color terahertz generation in plasma gas targets: energy and pressure dependence, Opt. Express 18, 15130 (2010).

[12] A. R. Niknam, M. R. Banjafar, F. Jahangiri, S. Barzegar, and R. Massudi, Resonant terahertz radiation from warm collisional inhomogeneous plasma irradiated by two gaussian laser beams, Phys. Plasmas 23, 053110 (2016).

[13] S. Safari, A. R. Niknam, F. Jahangiri, and B. Jazi, Terahertz radiation generation through the nonlinear interaction of hermite and laguerre gaussian laser beams with collisional plasma: Field profile optimization, J. Appl. Phys. 123, 153101 (2018).

[14] F. Jahangiri, M. Hashida, S. Tokita, T. Nagashima, K. Ohtani, M. Hangyo, and S. Sakabe, Directional terahertz emission from air plasma generated by linearly polarized intense femtosecond laser pulses, Appl. Phys. Express 5, 026201 (2012).

[15] G. L. Carr, M. C. Martin, W. R. McKinney, K. Jordan, G. R. Neil, and G.P. Williams, High-power terahertz radiation from relativistic electrons, Nature (London) 420, 153 (2002).

[16] S. Bielawski, C. Evains, T. Hara, M. Hosaka, M. Katoh, S. Kimura, A. Mochihashi, M. Shimada, C. Szwaj, T. Takashi, and Y. Takashima, Tunable narrowband terahertz emission from mastered laserelectron beam interaction, Nat. Phys. 4, 390 (2008).

[17] A.-S. Muller, Reviews of Accelerator Science and Technology (World Scientific Publishing Company, Singapore, 2010) Chap. Accelerator-Based Sources of Infrared and Terahertz Radiation.

[18] N. Stojanovic and M. Drescher, Accelerator- and laserbased sources of high-field terahertz pulses, J. Phys. B 46, 192001 (2013).

[19] P. Salen, M. Basini, S. Bonetti, J. Hebling, M. Krasilnikov, A. Y. Nikitin, G. Shamuilov, Z. Tibai, V. Zhaunerchyk, and V. Goryashko, Matter manipulation with extreme terahertz light: Progress in the enabling thz technology, Phys. Rep. 836-837, 1 (2019).

[20] A.-S. Muller and M. Schwarz, Synchrotron Light Sources and Free-electron Lasers (Springer International Publishing Switzerland, 2015) Chap. Accelerator-Based $\mathrm{THz}$ Radiation Sources.

[21] A. Gover, R. Ianconescu, A. Friedman, C. Emma, N. Sudar, P. Musumeci, and C. Pellegrini, Superradiant and stimulated-superradiant emission of bunchedelectron beams, Rev. Mod. Phys. 91, 035003 (2019).
[22] E. Hemsing, G. Stupakov, D. Xiang, and A. Zholents, Beam by design: Laser manipulation of electrons in modern accelerators, Rev. Mod. Phys. 86, 897 (2014).

[23] I. Ben-Zvi, L. F. D. Mauro, S. Krinsky, M. G. White, and L. H. Yu, Proposed uv FEL user facility at BNL, Nucl. Instrum. Methods Phys. Res., Sect. A 304, 181 (1991).

[24] L. H. Yu, Generation of intense uv radiation by subharmonically seeded single-pass free-electron lasers, Phys. Rev. A 44, 5178 (1991).

[25] E. Allaria and G. D. Ninno, Soft-X-ray Coherent Radiation Using a Single-Cascade Free-Electron Laser, Phys. Rev. Lett. 99, 014801 (2007).

[26] G. Stupakov, Using the beam-echo effect for generation of short-wavelength radiation, Phys. Rev. Lett. 102, 074801 (2009).

[27] D. Xiang and G. Stupakov, Enhanced tunable narrow-band thz emission from laser-modulated electron beams, Phys. Rev. ST Accel. Beams 12, 080701 (2009).

[28] S. Kumar, D. Kim, and H. Kang, Tunable thz radiation generation using density modulation of a relativistic electron beam, Nucl. Instrum. Methods Phys. Res., Sect. A 729, 19 (2013).

[29] M. Dunning, C. Hast, E. Hemsing, K. Jobe, D. McCormick, J. Nelson, T. O. Raubenheimer, K. Soong, Z. Szalata, D. Walz, S. Weathersby, and D. Xiang, Generating Periodic Terahertz Structures in a Relativistic Electron Beam through Frequency Down-Conversion of Optical Lasers, Phys. Rev. Lett. 109, 074801 (2012).

[30] Z. Wang, D. Huang, Q. Gu, Z. Zhao, and D. Xiang, Echoenabled tunable terahertz radiation generation with a lasermodulated relativistic electron beam, Phys. Rev. ST Accel. Beams 17, 090701 (2014).

[31] H. Zhang, W. Wang, S. Jiang, C. Li, Z. He, Q. Jia, L. Wang, and D. He, Generation of frequency-chirped density modulation electron beam for producing ultrashort thz radiation pulse, Phys. Rev. Accel. Beams 23, 020704 (2020).

[32] D. Gordon, C. E. Clayton, T. Katsouleas, W. B. Mori, and C. Joshi, Microbunching of relativistic electrons using a two-frequency laser, Phys. Rev. E 57, 1035 (1998).

[33] M. Kumar and V. K. Tripathi, Terahertz radiation from a laser bunched relativistic electron beam in a magnetic wiggler, Phys. Plasmas 19, 073109 (2012).

[34] S. C. Sharma, J. Panwar, and R. Sharma, Modeling of terahertz radiation emission from a free-electron laser, Contrib. Plasma Phys. 57, 167 (2017).

[35] A. A. Zholents and M. S. Zolotorev, Femtosecond X-ray Pulses of Synchrotron Radiation, Phys. Rev. Lett. 76, 912 (1996).

[36] M. Kozk, N. Schnenberger, and P. Hommelhoff, Ponderomotive Generation and Detection of Attosecond FreeElectron Pulse Trains, Phys. Rev. Lett. 120, 103203 (2018).

[37] C. Zhou, Y. Bai, L. Song, Y. Zeng, Y. Xu, D. Zhang, X. Lu, Y. Leng, J. Liu, Y. Tian, R. Li, and Z. Xu, Direct mapping of attosecond electron dynamics, Nat. Photonics 15, 216 (2021).

[38] K. Holldack, S. Khan, R. Mitzner, and T. Quast, Femtosecond Terahertz Radiation from Femtoslicing at Bessy, Phys. Rev. Lett. 96, 054801 (2006). 
[39] R. C. Davidson, Physics of Nonneutral Plasmas, 2nd ed. (World Scientific Publishing Company, Singapore, 2001).

[40] S. Kamada, T. Yoshida, and T. Aoki, The chirp-control of frequency-tunable narrowband terahertz pulses by nonlinearly chirped laser pulse beating, Appl. Phys. Lett. 104, 101102 (2014).

[41] H. Zhang, W. Wang, S. Jiang, C. Li, Z. He, S. Zhang, Q. Jia, L. Wang, and D. He, Chirp control of tunable terahertz synchrotron radiation, Opt. Lett. 45, 4674 (2020).
[42] Z. Tibai, G. Toth, M. I. Mechler, J. A. Fulop, G. Almasi, and J. Hebling, Proposal for Carrier-Envelope-Phase Stable Single-Cycle Attosecond Pulse Generation in the ExtremeUltraviolet Range, Phys. Rev. Lett. 113, 104801 (2014).

[43] W. Kruer, The Physics of Laser Plasma Interactions (CRC Press, Boca Raton, 2002).

[44] M. Rezaei-Pandari, M. Akhyani, F. Jahangiri, A. R. Niknam, and R. Massudi, Effect of temporal asymmetry of the laser pulse on electron acceleration in vacuum, Opt. Commun. 429, 46 (2018). 